\title{
An Efficient Image Retrieval System using GLCM Features and Kullback-Leibler Divergence
}

\author{
Ch. Kodanda Ramu, T. Sita Mahalakshmi
}

\begin{abstract}
Image processing is a process of extracting features from an image. The features of the image are extracted using the correlation model, based on Gray-Level Co-Occurrence Matrix (GLCM). Each of the images considered for data set are converted into gray level before applying Gaussian Mixture Model (GMM). The features extracted from GLCM are given as an input to the model-based technique so that the relative Probability Density Functions (PDF) are extracted. The comparison is carried out in the same manner by identifying the relative PDF of the training set and test data by using KullbackLeibler divergence method (KL-Divergence). In this paper an attempt is made for developing an effective model to retrieve the images based on features by considering GLCM and GMM. The results derived show that the proposed methodology is able to retrieve images more effectively.
\end{abstract}

Keywords: GLCM, GMM, PDF, Correlation, KL-Divergence, MSE, RMSE, PSNR, IF.

\section{INTRODUCTION}

As the number of images accessible in the global scenario are increasing exponentially, the area of image processing turned to be more prevailing and its importance is more focused during the retrieval of specific images of interest. This area has become more dominant as its applications are enhancing from ordinary images to medical images. The other applications of this field include content-based retrieval, medical analysis, video retrievals etc.

In this paper, a methodology is proposed by considering an application of retrieval using the content. In order to extract the contents, features play a crucial role. GLCM is considered for the effective identification of the features. Among the various features related to the retrieval of the images based on GLCM, we have considered the correlation model. The features from the image is extracted by using GLCM methodology and each of these features are given as an input to the model, based on Gaussian distribution. Then the relative Probability Density Functions (PDF) of the images are extracted and the PDF of the entire image data set are estimated and are stored in the database. The same analogy is carried out for the test data.

In the current methodology, we have taken the images from MirFlikr data set. The correlation of the images are identified using KL-Divergence method.

Revised Manuscript Received on February 05, 2020.

* Correspondence Author

Ch. Kodanda Ramu*, research scholar in CSE Department, GITAM (Deemed to be university), Vizag, Andhra Pradesh, India.

Dr. T. Sita Mahalakshmi, professor in the CSE Department, GITAM (Deemed to be University), Vizag, Andhra Pradesh, India.

(C) The Authors. Published by Blue Eyes Intelligence Engineering and Sciences Publication (BEIESP). This is an open access article under the CC BY-NC-ND license (http://creativecommons.org/licenses/by-nc-nd/4.0/)
Most of the articles related to this area of research witness the methodologies, where the identification of the relevant images from the image pool is considered by using different feature methodologies.

However, the features considered for the retrieval of the relevant images are appropriate to retrieve the images more accurately, due to several associated factors namely dimension, colour, homogeny, intensity, texture, and correlation. The present methodologies in the literature are confined to some of the feature extraction and are also based on edges or shape. However, to identify the images more appropriately, the correlation between the images together with the statistical properties of the images needs to be considered. This present model makes an attempt in this direction.

\section{RELATED WORK}

In order to retrieve the relevant images from the data sets several methods have been presented in the literature of which methodologies based on edge and boundary extractions are mostly verified [1] [2].

However, the limitation of these methods is that if the shape of the image changes, the retrieval accuracy is at stake. In order to overcome this challenge methodologies based on neural networks, Singular Value Decomposition (SVD), pattern matching algorithms, bio-inspired technologies are also presented in the literature [3], [4], [5], [6], [7], and [8]. However, to retrieve the images of interest more accurately it is necessary to identify the features.

Therefore, feature extraction is important in the extraction of the images more optimistically. Most of the works are highlighted in this direction [9], [10], [11], [12]. However these methodologies are highlighted by considering the nonparametric model-based approaches.

Since the non-parametric models are considered, the retrieval accuracy cannot be assured due to the fact that the non-parametric approaches consider only certain features and hence the efficient retrieval cannot be achieved.

In contrary, the model-based approaches considered the parameters inside the image regions and these parameters are considered as the features and the retrieval of the images are assumed to be more efficient. Hence parametric based approach is utilized by considering the GMM and the parameters of the GMM along with the features derived by using the GLCM model.

This paper is structured as follows, in section 3 of the article, the Data set considered is presented, the GMM is highlighted in section 4, and the methodology together with GLCM is presented in section 5 of the article. The KLDivergence method is discussed in section 6 of this paper, and in section 7, the experimental results are highlighted. 
The evaluations of the derived results are presented in the section 8 and the article is concluded in the section 9 .

\section{IMAGE DATASET}

MirFlikr data set is considered for the experimentation carried out in this model. This data set consists of $11,00,426$ images of different objects. Among these objects around 35,000 objects are tagged with the image information. The sample image data set is presented in figure.1

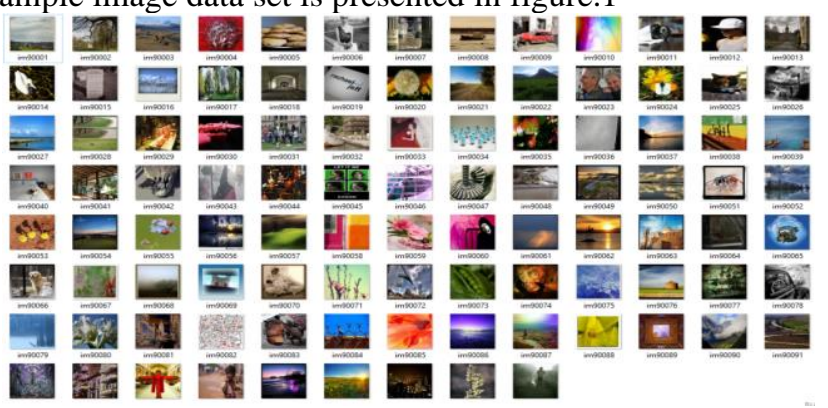

Figure1:Sample Image Data set

\section{GAUSSIAN MIXTURE MODEL (GMM)}

GMM is used in [12] [13] for image segmentation. This includes the GMM as a special case. The Probability Density Function of the GMM is given as follows

$$
N(x, \mu, \sigma)=\frac{1}{\sigma \sqrt{2 \prod}} e^{-\frac{(x-\mu)^{2}}{\sigma^{2}}}
$$

Where $\mathrm{x}$ is our data points, The $\mu$ and $\sigma$ are mean and variance of GMM respectively.

The GMM is considered because it segments the images which are both symmetric and asymmetric in nature. GMM is based on the probabilistic model which molds the data which is generated from image data points from a combination of Gaussian distributions with number of unidentified parameters. One might think that hybrid models circulate a compilation of $\mathrm{k}$ - means to include information about the variation in the data structure, as well as the underlying Gaussian centers.

\section{METHODOLOGY}

In order to present the model, the following steps are to be considered.

Step 1: Consider the image from the data set

Step 2: Convert into Gray scale image.

Step 3: Identify the correlation features using GLCM.

Step 4: Extract the PDF's using GMM.

Step 5: Repeat the steps 2 to 4 steps for the testing set also.

Step 6: Compare the training set images with the test images and the images retrieved are compared for accuracy using KL-Divergence method as shown in figure. 2

Step 7: The results derived are evaluated using Image Quality Metrics.

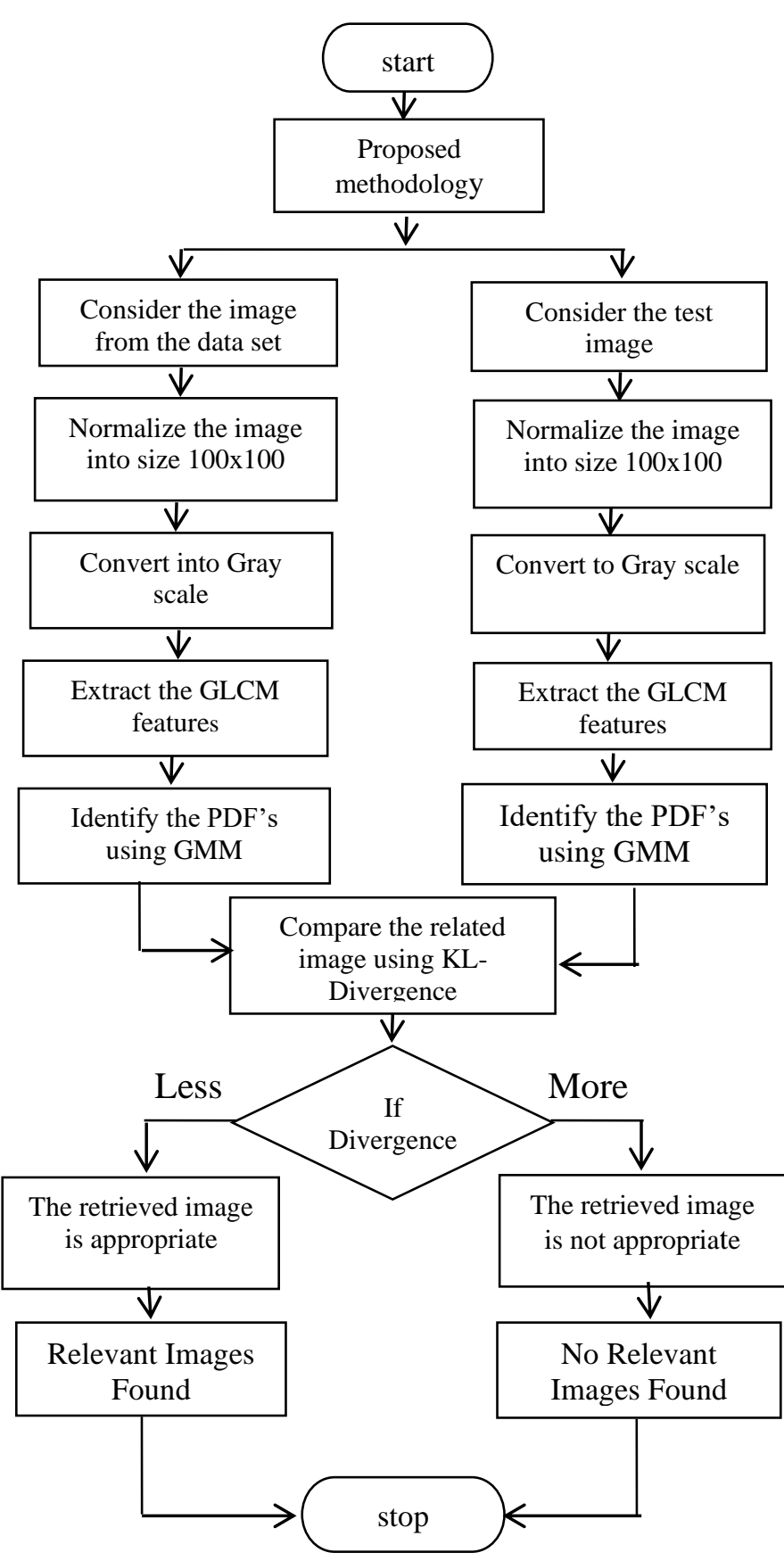

Figure2: Flow Chart of the proposed model

\section{GRAY-LEVEL CO-OCCURRENCE MATRIX}

The arithmetical technique of examining texture that considers the spatial correlation of pixels is the GLCM [14]. This function will describe the image by taking the texture and by calculating the pixel values and spatial coordinates meet in the image, creating a GLCM, and then extracting statistical measures from this matrix. An image composed of pixels each with an intensity, the GLCM is a tabulation of how often different combinations of gray levels co-occur in an image or image section. 


\section{KL-DIVERGENCE}

Kullback-Leibler divergence( KL-Divergence)[15] is used to identify the correlation between the acquired image and the images in the data set and also to measure the difference between different probability distributions. The formula for calculating the KL Divergence is given by

$$
D_{K L}(p(x) \| q(x))=\sum_{x \in X} p(x) \ln \frac{p(x)}{q(x)}
$$

Where $\mathrm{p}(\mathrm{x})$ represents the relative PDF of the training set image , $\mathrm{q}(\mathrm{x})$ represents the relative PDF of the test set image.

\section{EXPERIMENTAL RESULTS}

The present article is implemented using Dot Net environment and the experimental outputs are shown in figure. 3

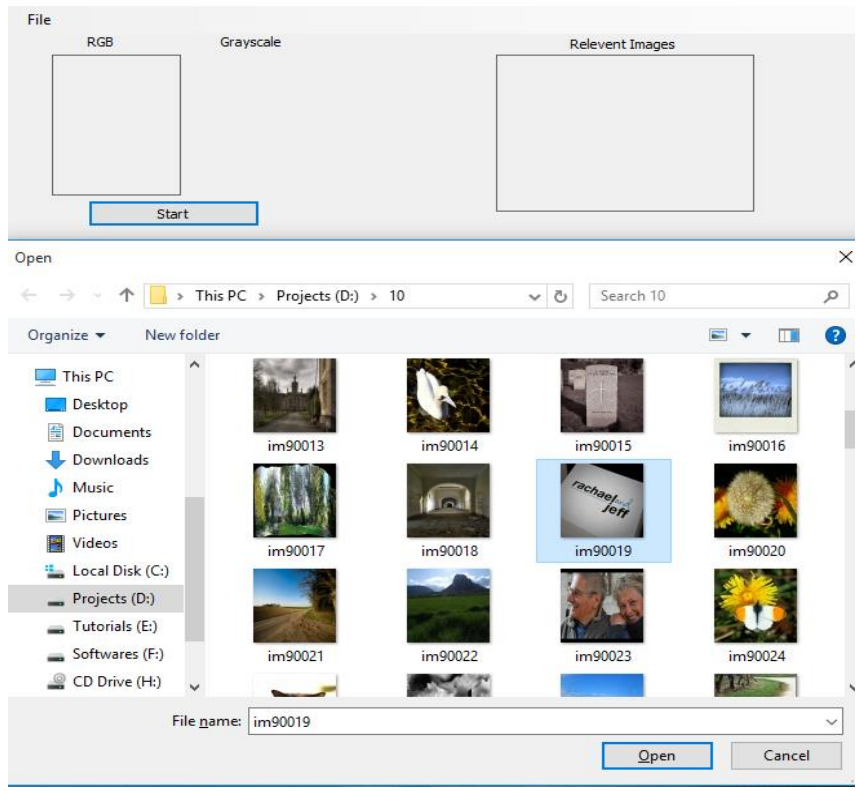

Figure3: Select the image from the folder

The selected color image is shown in Figure.4 and the equivalent gray scale image is shown in Figure.5.The search results are shown in Figure.6 and Figure.7

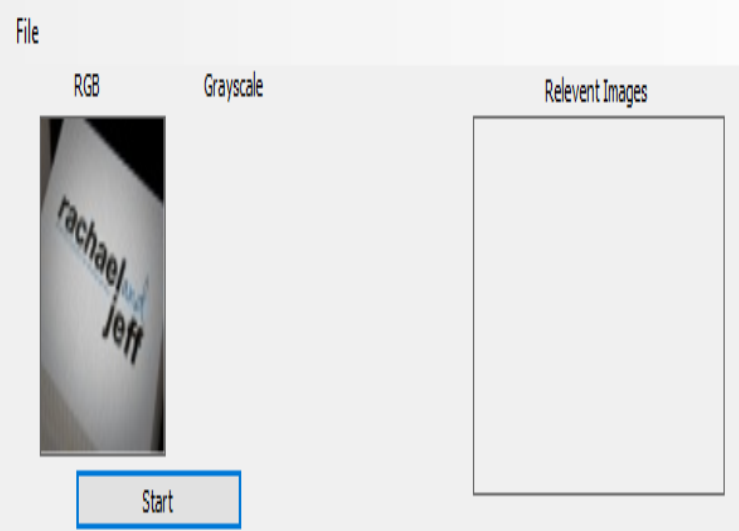

Figure 4: Selecting RGB image

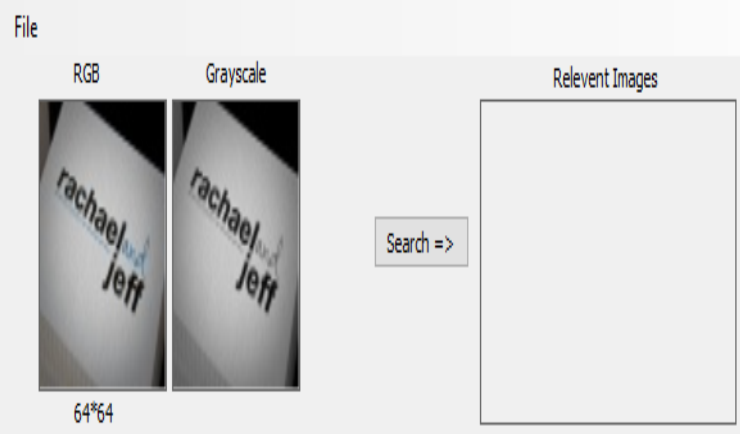

Figure 5: Convert RGB to Grey scale

File
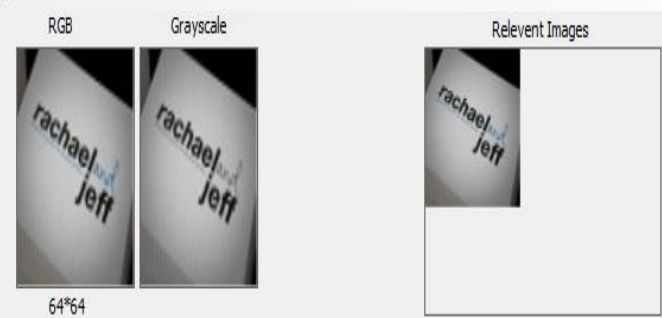

Figure 6: Relevant image search from the database

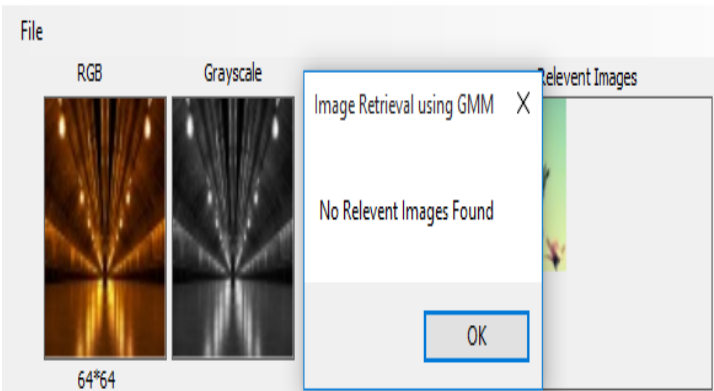

Figure 7: Image is not found

\section{EVALUATION}

In order to evaluate the present model,it is considered to check the similarity between test image and retrieved image. We have considered four metrics namely Mean squared error (MSE), Root Mean Square Error (RMSE), Peak Signal to noise Ratio (PSNR) and Image Fidelity (IF). First of all it is imperative to find out the pixel error between test image and retrieved image according to the image data discrepancy. The results of the above metrics are presented in table. 1

Mean Squared Error (MSE): which is a statistical function,it is defined as the square of differences in the pixel values between the corresponding pixels of the two images. This function take as input two grayscale images of same size and output a single numerical Value. It is commonly used to evaluate the effectiveness of image. The equation for calculating the MSE is given bellow

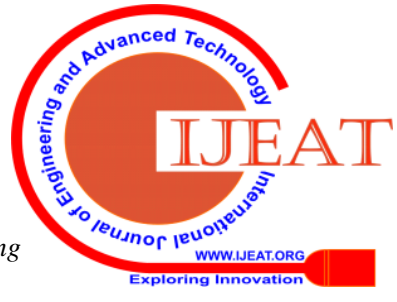




$$
M S E=\frac{1}{M N} \sum_{x=0}^{M-1} \sum_{y=0}^{N-1}[f(x, y)-\widehat{f}(x, y)]^{2}
$$

Where $\mathrm{M} \& \mathrm{~N}$ are number of rows and columns of the input image matrix and $\mathrm{x}, \mathrm{y}$ represents row number and column number of the pixel value in the image matrix. Here $\mathrm{f}$ is the test image matrix and $\mathrm{f}^{\wedge}$ is the retrieved image matrix .

Root Mean Square Error (RMSE): which is a function, it takes an input two grayscale images of same size $\mathrm{M}^{*} \mathrm{~N}$ and output a single floating point value. It is a numeric method for computing the difference between two images. Efficiency of the Image.Reconstruction algorithms is evaluated by computing the pixel wise difference between original and reconstructed image. We will use RMSE to compare our test image with the retrieved image.

$$
R M S E=\sqrt{\frac{\sum_{x=0}^{M-1} \sum_{y=0}^{N-1}[f(x, y)-\widehat{f}(x, y)]^{2}}{M * N}}
$$

Where $\mathrm{M} \& \mathrm{~N}$ are number of rows and columns of the input image matrix and $\mathrm{x}, \mathrm{y}$ represents row number and column number of the pixel value in the image matrix. Here $f$ is the test image matrix and $\mathrm{f}^{\wedge}$ is the retrieved image matrix.

Peak Signal to Noise Ratio (PSNR): PSNR is used to determine the relationship between the maximum signal strength and the power of distorted noise, which affects accuracy. The peak error between the test image and the received image is measured in terms of PSNR. A higher PSNR indicates a higher image quality.

$$
P S N R=10 \log _{10}\left(\frac{R^{2}}{M S E}\right)
$$

Where $\mathrm{R}$ is the maximum deviation of the type of input image data. Here $\mathrm{R}^{2}$ is the maximum allowed value for a pixel. In the case where one byte image of the signal per pixel per channel is 255 . When the two images are identical, the MSE will give zero, which will lead to an invalid division by zero in the PSNR formula. In this case, the PSNR is not determined, and this problem should be addressed separately.

Due to the pixel values in an image having very wide dynamic range, logarithmic scale is done.

Image Fidelity (IF): this is the process which display the ability of an image accurately without any visible distortion or loss of information. When there is no difference obtained between test image and retrieved image, then we can say that there is no loss in retrieved image.

$$
I F=1-\frac{\sum_{x=0}^{M-1} \sum_{y=0}^{N-1}[f(x, y)-\hat{f}(x, y)]^{2}}{\sum_{x=0}^{M-1} \sum_{y=0}^{N-1}[f(x, y)]^{2}}
$$

Where $\mathrm{M} \& \mathrm{~N}$ are number of rows and columns of the input image matrix and $\mathrm{x}, \mathrm{y}$ represents row number and column number of the pixel value in the image matrix. Here $f$ is the test image matrix and $\mathrm{f}^{\wedge}$ is the retrieved image matrix.

The simulation is best illustrated with the performance analysis against the test Image and the image which is extracted. The mean square error is gradually decreased with root mean square error values likewise surge in the data.
This is illustrated with the ten sample images. The value of PSNR is also being found to be varying along with the IF having touch fluctuating values. IF validation should be effectively and consistently increasing and with each samples the variations are under acceptable levels ranging from 0.3 to 0.7 for more informative sample.

Table 1: Performance of evaluation

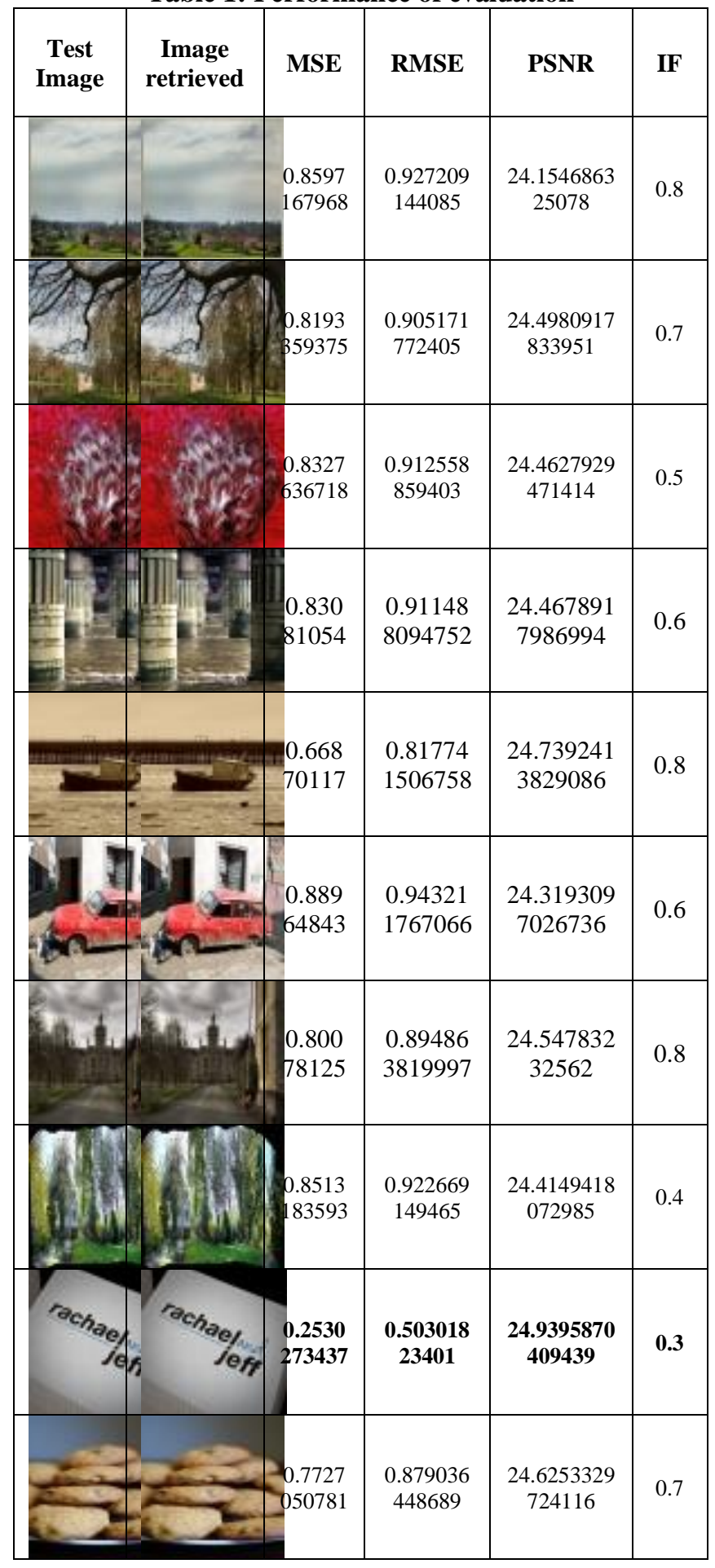

\section{CONCLUSION}

In this paper an attempt is made for developing an effective model to retrieve the images based on features by considering GLCM and GMM. 
The results derived shows that the proposed methodology is able to retrieve images more effectively. The performance evaluation of the model is shown in table. 1 It can be observed that the retrieved images are nearer to the test images and the values of MSE, RMSE, PSNR and IF validate the same. The MSE and RMSE for all the images retrieved is nearer to 0 and the PSNR values are positive and high, and IF values are also nearer to 0 . This shows that the proposed model is able to retrieve the images more effectively. This methodology can be applied to situations in particular, where the images need to be retrieved from huge data sets based on the content.

\section{REFERENCES}

1. Tiecheng Liu, Song Wang and Feng Ge, "Evaluating Edge Detection through Boundary Detection", EURASIP JASP, Volume 2006, Article ID 76278, Pages 1-15.

2. Xiaolin Wu, Paul Bao and Lei Zhang. "Canny Edge Detection Enhancement by Scale Multiplication”. IEEE TRANSACTIONS ON PAMI, SEPTEMBER 2005, VOL. 27, NO. 9.

3. Rowayda A. Sadek "SVD Based Image Processing Applications: State of The Art, Contributions and Research Challenges" AASTMT, Vol. 3, No. 7, 2012.

4. Tarek Z, Ahmed E "Automated Steel Bridge Coating Inspection using Neural Network Trained Image Processing", Concordia University, Montréal, Québec, Canada H3G 2W, Jan 2019.

5. Deep Suman Dev1 and Dakshina Ranjan Kisku2"Improved Pattern Matching Algorithm”, Appl. Math. Inf. Sci. 2017, 11, No. 4, 11631184.

6. Freund R, Osuna E and Girosi F, "Training Support Vector Machines: An Application to Face Detection", Proc. IEEE Conf. CVPR, pp. 130-136, 1997.

7. Kirti T and Deepak R, "Bio-Inspired Optimization Techniques - A Critical Comparative Study”. July 2013 Volume 38 Number 4.

8. Noorhayati Mohamed Noor, Norharyati Md Ariff1, Noor Elaiza Abdul Khalid, Saadiah Yahya, "A Review of Bio-inspired Algorithms as Image processing Techniques".

9. Suresh A "Image Texture Classification using Gray Level CoOccurrence Matrix Based Statistical Features", Vol.75, Issue 4 (2012), pp. 591-597.

10. Miguel Vega, Salvador Vilene, "Using the Kullback-Leibler Divergence to Combine Image Priors in Super-Resolution Image Reconstruction", IEEE 17th ICIP, September 2010, pp. 26-29.

11. LiCao, Zhihua Ban and Jianguo "Super pixel Segmentation Using Gaussian Mixture Model, IEEE transactions on IP, 2018, vol. 27, no. 8.

12. Shilpa Hat war "GMM based Image Segmentation and Analysis of Image Restoration Techniques", January 2015, Vol 109, No. 16.

13. Zarpak, Behnam, Yari, Gholamhossein \& Farnoosh, R. "Image Segmentation using Gaussian Mixture Models". IUST IJES-19.

14. P. Maharajah "Image Texture Feature Extraction Using GLCM Approach", IJSRP, 2013, Volume 3, Issue 5.

15. Muhammad Hanif "An Effective Image Restoration Using Kullback-Leibler Divergence Minimization, 2014 IEEE.

\section{AUTHORS PROFILE}

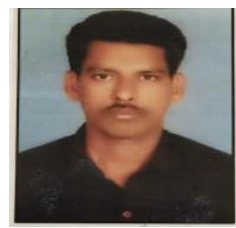

Ch. Kodanda Ramu is a research scholar in CSE Department, GITAM (Deemed to be university), Vizag, Andhra Pradesh, India. He received M.Tech in 2010. His research interests are Image Mining, Image Processing, Digital Forensic and Computer Networks.

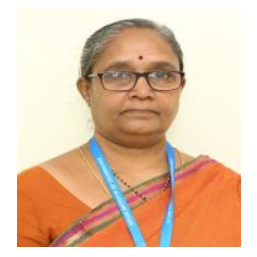

Dr. T. Sita Mahalakshmi is a professor in the CSE Department, GITAM (Deemed to be University), Vizag, Andhra Pradesh, India. She has published 20 research papers in National and International journals and presented papers at various conferences, seminars and workshops. Her current research interests are Image Processing, Image Mining, Data Mining and Computer Networks. 